\title{
MODELING OF DNA TRANSPORT IN VISCOELASTIC ELECTRO-HYDRODYNAMIC FLOWS FOR ENHANCED SIZE SEPARATION
}

\author{
B. Chami ${ }^{1}$, M. Socol ${ }^{1}$, M. Manghi ${ }^{2}$, and A. Bancaud ${ }^{1 *}$ \\ ${ }^{1}$ LAAS-CNRS, Toulouse, France \\ ${ }^{2}$ Laboratoire de Physique Théorique (IRSAMC), Université de Toulouse, CNRS, UPS, France
}

* Correspondence should be sent to:

Dr. Aurélien BANCAUD

LAAS-CNRS, 7 avenue du colonel Roche, BP 54200, 31031 Toulouse Cedex, France

Tel : 0033561336246

Email : abancaud@,laas.fr 


\begin{abstract}
WORDS)
DNA separation and analysis has advanced over the last years benefiting from microfluidic systems that reduce sample volumes and analysis costs, essential for sequencing and disease identification in body fluids. We recently developed the $\mu$ LAS technology that enables the separation, concentration, and analysis of nucleic acids with high sensitivity. The technology combines a hydrodynamic flow actuation and an opposite electrophoretic force in viscoelastic polymer solutions. Combining hydrodynamic first principles and statistical mechanics, we provide, in this paper, a quantitative model of DNA transport capable of predicting the device performance with the exclusive use of one adjustable parameter associated to the amplitude of transverse viscoelastic forces. The model proves to be in remarkable agreement with DNA separation experiments, and allows us to define optimal conditions that result in a maximal resolution length of $7 \mathrm{bp}$. We finally discuss the usefulness of our model for separation technologies involving viscoelastic liquids.
\end{abstract}

\title{
KEYWORDS
}

DNA separation, viscoelasticity, transverse migration, resolution length, DNA transport 


\section{INTRODUCTION}

Microfluidic technologies have a demonstrated potential for processing nucleic acids (Liu and Mathies, 2009; Paegel et al., 2003). High efficiency separation of DNA, which is required for a wide variety of applications, including sequencing and molecular diagnostics, is principally achieved with capillary electrophoresis (CE) systems (Albarghouthi and Barron, 2000). Commercial CE instruments however typically have a maximum sensitivity of $\sim 100 \mathrm{pg} / \mu \mathrm{L}$, requiring the integration of an on-line sample concentration module, which consists in stacking the sample in a region associated to a sudden reduction in DNA mobility. The standard technique, coined electrokinetic stacking (Simpson et al., 2008), exploits the interface between low- and high-conductivity buffers in order to abruptly slow down DNA migration. DNA concentration has also been achieved without changes in buffer composition using concentration gradients of separation matrices (Kuo et al., 2003). In the case of homogeneous buffer and polymer solutions, it has been proposed to convey DNA molecules with a hydrodynamic flow and to apply a counter electric field only in a sub-section of a capillary (Astorga-Wells et al., 2007, 2005; Park and Swerdlow, 2003). There is therefore a gradient in electrophoretic mobility along the separation channel, and the amplitude of the electric field is set to stall the progression of DNA and trigger its accumulation. The same concept has been investigated with electro-osmotic flows in balance with counter hydrodynamic flows to concentrate microparticles in a microfluidic chip with a constriction(Lettieri et al., 2003). Finally, the temperature gradient focusing (TGF) technique (Ross and Locascio, 2002) also consists in balancing the electrophoretic transport of DNA with the flow of the solution using an electrophoretic velocity gradient associated to temperature gradient (Ge et al., 2015).

We recently described the $\mu \mathrm{LAS}$ technology, standing for $\mu$-laboratory DNA Analysis and Separation, that simultaneously separates, enriches, and sizes DNA fragments within minutes (Ranchon et al., 2016). It consists in maneuvering DNA migration in a viscoelastic fluid using electro-hydrodynamic bi-directional actuation. The shearing of the liquid around the DNA molecules creates transverse elastic forces oriented towards the channel walls. These forces increase with the molecular weight (MW) of the DNA fragment, allowing matrix-free size separation. Furthermore, when operated in a channel with a funnel geometry, the amplitude of the transverse force is spatially modulated, generating a gradient in DNA mobility (Ranchon et al., 2016). Hence, with a continuous flow of material, $\mu$ LAS can also be used to perform DNA enrichment. The detection limit of this technology is $10 \mathrm{fg} / \mu \mathrm{L}$ for a fragment of $1000 \mathrm{bp}$ with a processing time of 20 min (Andriamanampisoa et al., 2018).

In this report, our goal is to derive a model of $\mu$ LAS operating principle in order to (i) enhance its performances, and (ii) develop a systems approach to integrate it in a fully automated Lab-on-Chip. For this, we perform an experimental and theoretical investigation of $\mu$ LAS for DNA analysis in the size range 50 to 1000 bp. We first report on an analytical model of particle transport in viscoelastic liquids under electrohydrodynamic actuation. This model allows us to predict the position, the width and resolution of DNA bands as a function of the macroscopic actuation parameters, microfluidic chip geometry, as well as the fluid properties. These predictions are in quantitative agreement with our experimental data, enabling us to define optimal settings for genotyping applications. The maximum resolution length of $7 \mathrm{bp}$ for a $50 \mathrm{bp}$ fragment and $30 \mathrm{bp}$ for a $500 \mathrm{bp}$ fragment appears to be insufficient for sequencing, but relevant for minute samples sizing. We finally discuss options to further enhance the resolution with the same technology.

\section{A FEW FACTS ON $\mu$ LAS TECHNOLOGY}

The design of the microfluidic chips, which are processed by silicon/glass micromachining (Ranchon et al., 2015), consists of a single straight channel with a funnel, as shown in Fig. 1A. We define $(O x)$ as the longitudinal axis along the flow direction, $(O y)$ along the channel width, and $(O z)$ along the channel height. The width $w(x)$ of the channel scales as $w_{0}\left(x / x_{0}\right)^{m}$ with $m$ the power law exponent describing of the constriction shape, $w_{0}$ and $x_{0}$ the minimal width and typical extension of the constriction, respectively. Our experiments consist in flowing a viscoelastic solution containing a DNA ladder sample by means of a pressure controller operating in the range 1 to 10 bar in either direction of the constriction. An electric field generated by a DC voltage supply operating in the range 0 to $400 \mathrm{~V}$ allows us to concurrently force DNA electrophoretic migration in the direction opposite to the flow. The readout of our experiments (Fig. 1B) is the fluorescence intensity distribution along the $(O x)$ axis, which is then used to measure the position and width of each resolved DNA band based on a Gaussian fit (Fig. 1C). Note that the reference position $x=0$ corresponds to the apex of the constriction. 
A
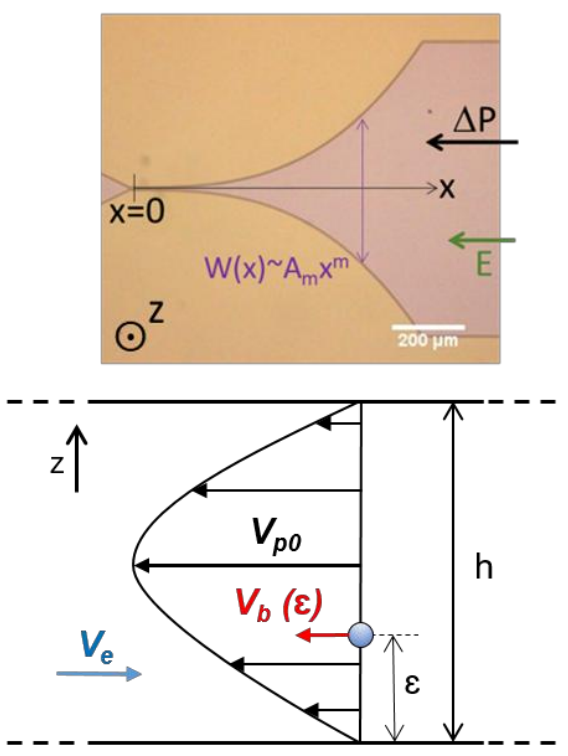

B
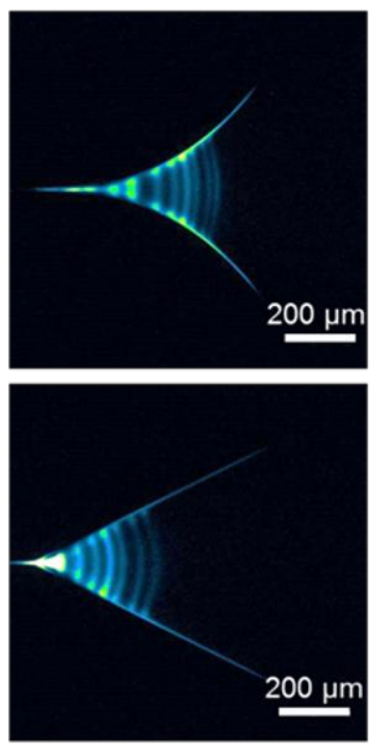
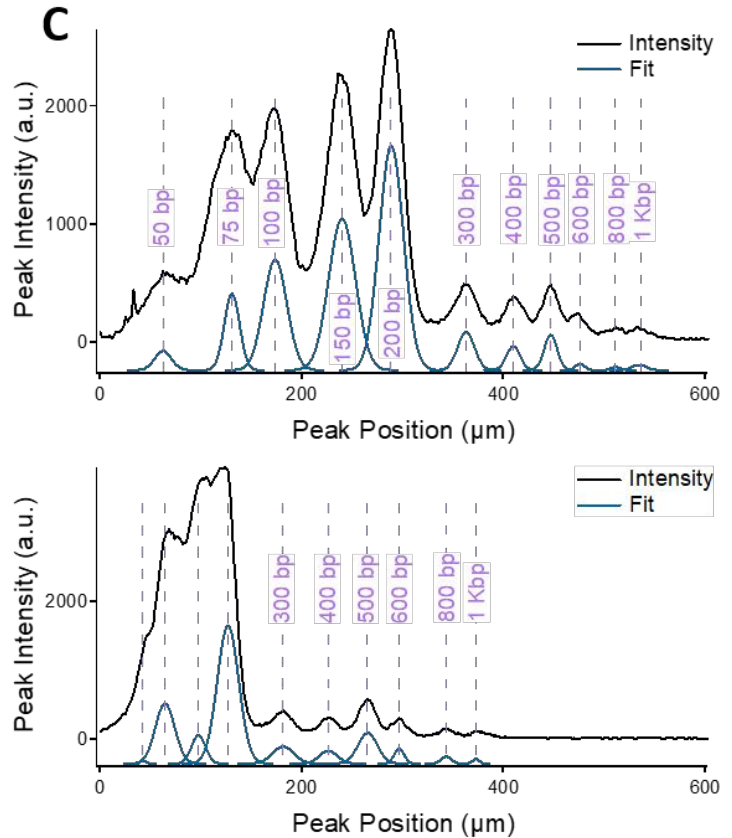

Figure 1: Principle of $\boldsymbol{\mu L A S}$ experiments. (A) The upper panel shows a bright field micrograph of a microchannel with a power-law profile ( $\mathrm{x}^{3}$-profile). The pressure and electric fields are actuated simultaneously in the same direction, but the negative charge of DNA induces an electrophoretic mobility in the opposite direction to the pressure flow. The sketch in the lower panel represents a bead at a vertical position $\varepsilon$ in a slit-channel of height $h$. The Poiseuille flow is characterized by

its maximum velocity $V_{p_{0}}$ and the electrophoretic velocity is $\mathrm{V}_{\mathrm{e}}$. The velocity of the particle is $\mathrm{V}_{\mathrm{b}}$. (B) The fluorescence micrographs of a $50 \mathrm{bp}$ DNA ladder separated in a chip with a power law $(\mathrm{m}=3)$ and linear $(\mathrm{m}=1)$ funnel profile(upper and

lower panel, respectively). The viscoelastic fluid is composed of $18 \%(\mathrm{~m}: \mathrm{w}) 40 \mathrm{kDa}$ PVP. The actuation pressure and voltage are set to $7 \mathrm{bar}$ and $300 \mathrm{~V}$, respectively, corresponding to maximum flow and electrophoretic velocities of 146 and $4.6 \mathrm{~mm} / \mathrm{s}$. (C) The fluorescence intensity profiles of the DNA ladder as a function of the position along the funnel is plotted in black. The fit with multiple Gaussian functions (see methods) is represented in blue. The respective size of each band is shown with purple vertical dashed lines.

\section{THE PHYSICAL MODEL}

Our goal is to express the resolution of DNA separation experiments as a function of the electric and hydrodynamic actuation parameters, DNA size, chip geometry, as well as fluid viscoelastic properties. For this, we derive an analytical expression of the transverse viscoelastic force exerted on spherical particles conveyed by electro-hydrodynamic actuation. We then use statistical physics to infer the distribution of tracers across the channel height, and come up with an expression that predicts the position, width, and resolution of DNA bands. These predictions are compared to experimental data in the following section.

\section{Expression of the transverse viscoelastic force with electro-hydrodynamic actuation}

For a particle of radius $a$ conveyed by a Poiseuille viscoelastic flow of maximum speed $V_{p_{0}}$ in a slit-like channel of height $h$ (Fig. 1A), the shearing of the fluid leads to an excess of elastic stress that results in transverse migration toward the channel centerline (D'Avino et al., 2017; Ho and Leal, 1976; Leshansky et al., 2007). For a Maxwell fluid, the transverse force $F_{T}$ reads:

$$
F_{T}(\varepsilon)=-2 \pi K \eta \tau a^{3} \frac{\partial \dot{\gamma}^{2}}{\partial z}(\varepsilon)
$$

with $\dot{\gamma}$ the shear rate, $\eta$ the viscosity, $\tau$ the fluid elastic relaxation time, and $\varepsilon$ the position of the particle. Note that according to the calculations of ref. (Ho and Leal, 1976), the proportionality factor $K$ is dependent on the polymer molecular weight and concentration.

Let us now consider the contribution of an electrophoretic velocity $V_{e}$ oriented opposite to the hydrostatic flow (Fig. 1A). Neglecting hydrodynamic interactions with the walls, the velocity of the bead is $V_{p}(\varepsilon)-V_{e}$ with $V_{p}(\varepsilon)$ the velocity of the fluid at position $\varepsilon$. The shear rate of the fluid on the upper side of the particle reads: 


$$
\dot{\gamma_{u}} \approx \frac{1}{\Delta z}\left\{V_{p}(\varepsilon+a+\Delta z)-\left[V_{p}(\varepsilon+a)-V_{e}\right]\right\}=\frac{d V_{p}}{d z}(\varepsilon+a)+\frac{\Delta z}{2} \frac{d^{2} V_{p}}{d z^{2}}(\varepsilon+a)+\frac{V_{e}}{\Delta z}
$$

with $\Delta z$ a typical small distance over which the shear rate is computed. We can then compute the difference of the square of the shear rate between the upper and lower sides of the particle:

$$
{\dot{\gamma_{u}}}^{2}-{\dot{\gamma_{b}}}^{2} \approx \frac{2}{\Delta z} \frac{d V_{p}}{d z}(\varepsilon)\left[2 V_{e}+\Delta z^{2} \frac{d^{2} V_{p}}{d z^{2}}(\varepsilon)\right]
$$

Assuming that the characteristic length scale of the flow around the bead $\Delta z$ is on the order of $a$, we deduce the expression of the transverse force:

$$
F_{T}^{e l}(\varepsilon)=-4 \pi K \eta \tau a \frac{d V_{p}}{d z}(\varepsilon)\left[2 V_{e}+a^{2} \frac{d^{2} V_{p}}{d z^{2}}(\varepsilon)\right]
$$

If $V_{e}$ is equal to zero, we note that Eq. (4) is equivalent to Eq. (1). By plugging the expression of the parabolic flow field, $V_{p}(z)=4 V_{p_{0}} \frac{z}{h}\left(1-\frac{z}{h}\right)$, in Eq. (4), we get:

$$
F_{T}^{e l}(\varepsilon)=-32 \pi K \eta \tau \frac{a}{h} V_{p_{0}}\left(V_{e}-4 V_{p_{0}} \frac{a^{2}}{h^{2}}\right)\left(1-\frac{2 \varepsilon}{h}\right)
$$

Therefore, the use of an electric field reverses the orientation of the transverse viscoelastic force. We note that electro-osmosis is neglected in our model, because our experiments are carried out with polymer with coating properties (Kaneta et al., 2006). If the particle is small $a \ll h$ and the electrophoretic velocity comparable to the hydrodynamic velocity, Eq. (5) can be simplified as:

$$
F_{T}^{e l}(\varepsilon)=-32 \pi K \eta \tau \frac{a}{h} V_{p_{0}} V_{e}\left(1-\frac{2 \varepsilon}{h}\right)
$$

Consequently, the transverse viscoelastic force with electro-hydrodynamic actuation scales linearly with the tracer size, hydrodynamic velocity, and electrophoretic velocity.

Using 3D finite-element simulations performed with COMSOL, we checked the validity of Eq. (6) for a 100 $\mathrm{nm}$ particle flowing in a $2 \mu \mathrm{m}$-deep channel. We assumed the fluid to be Newtonian and set the maximum fluid velocity $V_{p_{0}}$ to $75 \mu \mathrm{m} / \mathrm{s}$. We used different electrophoretic velocities spanning 1.5 to $25 \mu \mathrm{m} / \mathrm{s}$ (Supplementary Fig. S1). In each condition, the particle was placed at different vertical positions, and we computed the difference between the square of the shear rate on the upper and lower apex of the particle, and confirmed that the simulation datasets could be rescaled with the electrophoretic velocity.

\section{Force close to the wall}

Eq. (6) has been computed for a particle located far from the walls, i.e. for a distance $\varepsilon \widetilde{>} 3 a$. We expect the transverse viscoelastic force to drop to zero at the wall in order to guarantee the continuity of the stress tensor. Consequently, as a first order approximation, we assume that the force linearly drops to zero at the contact. The expression of the force near the wall then reads:

$$
F_{T_{\text {wall }}}^{e l}(\varepsilon) \simeq-32 \pi K^{\prime} \eta \tau \frac{a}{h} V_{p_{0}} V_{e} \frac{\varepsilon}{h} \equiv-k \varepsilon
$$

with $K^{\prime}=K h / l$ is a dimensionless constant with $l$ in the typical distance (of a few $a$ ) over which the force vanishes. This response is therefore analogous to a spring of elastic constant $k$ retaining the sphere toward the wall. We find the average position as a function of the local electrophoretic and hydrodynamic velocities as follows:

$$
\langle z\rangle=\frac{\int_{0}^{\infty} \varepsilon e^{-k \varepsilon^{2} /\left(2 k_{B} T\right)} d \varepsilon}{\int_{0}^{\infty} e^{-k \varepsilon^{2} /\left(2 k_{B} T\right)} d \varepsilon}=\sqrt{\frac{2 k_{B} T}{\pi k}}
$$

The variance (or second order moment) $\left\langle\delta z^{2}\right\rangle=\left\langle z^{2}\right\rangle-\langle z\rangle^{2}$ can be evaluated similarly to be $\left\langle\delta z^{2}\right\rangle=$ $\langle z\rangle^{2}(\pi / 2-1)$.

\section{Prediction for the position, width, and resolution}


In the following, we propose to model DNA chains of small MW of less than $1000 \mathrm{bp}$ as spheres of effective radius $a$. The link between $a$ and DNA MW will be discussed in the experimental section.

\section{Position}

The position of arrest of DNA corresponds to a situation of balance between hydrodynamics and electrophoresis:

$$
\frac{4 V_{p_{0}}}{h^{2}}\langle z\rangle(h-\langle z\rangle)=V_{e}
$$
for:

Plugging Eq. (8) into Eq. (9) in the limit of $\langle z\rangle \ll h$, we deduce that the conditions of arrest are achieved

$$
\frac{k_{B} T}{\pi^{2} K^{\prime} \eta \tau a}=\frac{V_{e}^{3}}{V_{p_{0}}}
$$

In order to reformulate this equation with global actuation parameters, we have to consider conservation laws for the hydrodynamic and electric field fluxes. Along the symmetry axis of the constriction, we have qualitatively:

$$
\left\{\begin{array}{l}
V_{p_{0}}(x)=V_{p_{\max }} \frac{w_{0}}{w_{0}+w_{0}\left(x / x_{0}\right)^{m}} \\
V_{e}(x)=V_{e_{\max }} \frac{w_{0}}{w_{0}+w_{0}\left(x / x_{0}\right)^{m}}
\end{array}\right.
$$

with $w_{0}$ the width of the channel at the apex $(x=0), x_{0}$ the typical extension of the constriction, and $V_{p_{\max }}$ and $V_{e_{\max }}$ the maximum hydrodynamic and electrophoretic velocities at the apex (see Fig. 1A). Assuming that the accumulation of DNA occurs far from the apex of the funnel, the denominator can be simplified, and the set of equations in (11) reinjected into (10) in order to define DNA position as a function of the fluid properties and actuation parameters (see Supplementary material for the expression of $V_{e_{\max }}$ and $V_{p_{\max }}$ as a function of the tension and pressure drop):

$$
\left(\frac{x_{D N A}}{x_{0}}\right)^{2 m} \simeq \pi^{2} K^{\prime} \frac{\eta \tau a}{k_{B} T} \frac{V_{e_{\max }}{ }^{3}}{V_{p_{\max }}}
$$

\section{Breadth}

Next, we propose to evaluate the breadth $B$ of a band along the longitudinal direction of the flow. We know that the vertical extension in which the particle diffuses corresponds to the second moment $\left\langle\delta z^{2}\right\rangle$. The typical time for a particle to travel across this region is $\tau_{T}=\sqrt{\left\langle\delta z^{2}\right\rangle} / V_{T}$ with $V_{T}$ the transverse velocity dictated by the viscoelastic force. During this time scale, particles can move along the flow direction due to the shear flow $\dot{\gamma}$. Hence, the width of the band $B$ is the result of the shear velocity, where we consider the same characteristic time $\tau_{T}$ for the movement of the DNA in the $x$ direction:

$$
B \approx \dot{\gamma} \sqrt{\left\langle\delta z^{2}\right\rangle} \tau_{T} \approx \dot{\gamma} \frac{\left\langle\delta z^{2}\right\rangle}{V_{T}}
$$
with:

Defining the transverse velocity as $V_{T}=k\langle z\rangle / 6 \pi \eta a$ and using fluctuation dissipation arguments, we end up

$$
B \approx \dot{\gamma} \frac{\left\langle\delta z^{2}\right\rangle^{2}}{\langle z\rangle} \frac{1}{D}
$$

where $D$ is the diffusion coefficient of the particle/DNA, taken to be equal to $k_{B} T / 6 \pi \eta a$. Given that the shear rate $\dot{\gamma}$ is equal to $4 V_{p_{0}} / h$, and plugging Eq. (8) and (10), we deduce: 


$$
B \approx \frac{3(\pi-2)^{2}}{32} \frac{h^{2}}{K^{\prime} \tau} \frac{1}{V_{p_{0}}}
$$

This expression can finally be reformulated as a function of the position of accumulation by using Eq. (11):

$$
B \approx \frac{3(\pi-2)^{2}}{32} \frac{h^{2}}{K^{\prime} \tau} \frac{1}{V_{p_{\max }}}\left(\frac{x_{D N A}}{x_{0}}\right)^{m}
$$

Finally, we can plug Eq. (12) in order to define the breadth as a function of global actuation parameters:

$$
B \approx \sqrt{\frac{\eta a h^{4}}{K^{\prime} k_{B} T \tau}}\left(\frac{V_{e_{\max }}}{V_{p_{\max }}}\right)^{3 / 2}
$$

Notably, this expression is independent of the shape of the constriction.

\section{Resolution per base pair}

Finally, with the definition of the position of the bands and their width, it is possible to compute the resolution, as defined as:

$$
\operatorname{Res}_{1,2}=\frac{2\left(x_{1}-x_{2}\right)}{B_{1}+B_{2}} \simeq \frac{2 \frac{\partial x}{\partial a}\left(\frac{a_{1}+a_{2}}{2}\right) \Delta a_{1,2}}{B_{1}+B_{2}} \simeq \frac{\frac{\partial x}{\partial a}\left(a_{1}\right) \Delta a_{1,2}}{B_{1}}
$$

with $x_{1}-x_{2}$ the distance between two consecutive bands and $B$ the width of each peak, and $\Delta a_{1,2}$ the difference in size between two consecutive DNA bands. After derivation of Eq. (12), this expression can be reformulated as:

$$
\frac{\operatorname{Res}_{1,2}}{\Delta a_{1,2}} \approx \frac{a_{1}{ }^{\left(\frac{1}{2 m}-1\right)}}{B_{1}} x_{0}\left[\frac{K^{\prime} \eta \tau}{k_{B} T} \frac{V_{e_{\max }}{ }^{3}}{V_{p_{\max }}}\right]^{\frac{1}{2 m}}
$$

The left term in Equation (19) is the resolution per base pair $\left(\mathrm{R}_{\mathrm{bp}}\right)$ (Heller, 2001), or the inverse of the resolution length (RSL) (Yamaguchi et al., 2015) which defines the minimal genomic distance between two consecutive peaks that can be separated with the technology. We can also plug the expression of the breadth $B_{1}$ using Eq. (17):

$$
R_{b p} \approx a_{1}\left(\frac{1}{2 m}-\frac{3}{2}\right) \frac{K^{\prime \frac{1}{2}(1 / m+1)} \eta^{\frac{1}{2}(1 / m-1)} \tau^{\frac{1}{2}(1 / m+1)}}{h^{2}} V_{e_{\max }}{ }^{\frac{3}{2}(1 / m-1)} V_{p_{\max }}{ }^{\frac{3}{2}(1-1 / 3 m)}
$$

According to equation (20), the resolution per base pair decreases with the size of the molecule, the viscosity of the solution, and the electrophoretic velocity, whereas it increases with the relaxation time of the solution and speed of the flow.

\section{MATERIALS AND METHODS}

\section{Chemicals and solutions}

The viscoelastic polymer solutions contained polyvinylpyrrolidone (PVP) of molecular weights (MW) 1.3 MDa or $40 \mathrm{kDa}$ purchased from Sigma Aldrich. The polymer powders were dissolved in 1X-TBE buffer (TrisBorate-EDTA (Sambrook et al., 1989)) during 1 day, and then supplemented with $2 \%$ (m:vol) Dithiothreitol (DTT, Sigma Aldrich) to prevent photodegradation. All solutions were filtered at $0.2 \mu \mathrm{m}$ pore size. The 50 $\mathrm{bp} /$ low range ladder DNA (Jena Bioscience) was diluted in the buffer at a final concentration spanning 0.2 to 1 $\mu \mathrm{mol} / \mathrm{L}$. The DNA was labeled with the intercalating dye YOYO-1 (Thermo-Scientific) at a dye to base pair ratio of 1:8.

\section{Chip fabrication and preparation for separation experiments}

The microfluidic chips were fabricated by DRIE etching of silicon, as described in ref. (Ranchon et al., 2016), through a homogeneous depth of $2 \mu \mathrm{m}$. The channel consisted of two head-to-head funnels with a power- 
law vs. linear profile (Fig. 1A). The minimal lateral dimension of the constriction $w_{0}$ was $5 \mu \mathrm{m}$, a dimension easily processed by photolithography. The microfluidic chip was first filled with ethanol to prevent the formation of bubbles. It was then washed with buffer, before introducing $45 \mu \mathrm{L}$ of DNA solution in the inlet and outlet reservoirs. The reservoirs were connected to a 7 bar MFCS Fluigent pressure controller, and to a DC voltage supply generating up to $400 \mathrm{~V}$ through a set of platinum electrodes.

\section{Data Acquisition and analysis}

The device was eventually placed on an inverted Zeiss microscope equipped with a 10X objective (Numerical Aperture=0.2), a sCMOS camera (Zyla Andor), and a light engine for cyan excitation (Lumencore). The experiment was performed using a set of ramps in pressure and voltage and by recording the build-up in fluorescence intensity at the constriction with an inter-frame interval of $100 \mathrm{~ms}$.

The collected videos were then analyzed by extracting the fluorescence intensity along the symmetry line of the funnel region ( $x$ arrow in Fig. 1). Intensity profiles were then processed to extract the position and width of each peak using the multipeak Gaussian-fitting package of Igor Pro.

\section{Rheological characterization of viscoelastic fluids}

Fluorescent beads (Bangs Labs) of diameter $2 a=1 \mu \mathrm{m}$ were diluted at a 1:5000 ratio in the target viscoelastic fluid. $20 \mu \mathrm{L}$ of this solution was then placed between two coverslips, and the motion of these particles was recorded at an acquisition rate of $1400 \mathrm{~Hz}$ using a cropped pixel array of $100 \mathrm{x} 100$ pixel $^{2}$. The objective was an oil immersion 40X (NA=1.4), and the corresponding pixel size was $162 \mathrm{~nm}$. Particle trajectories were extracted using the tracking algorithm TrackMate ${ }^{[18]}$ in order to retrieve the mean square displacement (MSD) dependence over time. Each measurement consisted of at least 5 independent trajectories. This data was finally analyzed assuming the viscoelastic solution behaved as a single relaxation time Maxwell fluid ${ }^{[19]}$ characterized by a linear viscosity $\eta$ and an elastic relaxation time scale $\tau$ (D'Avino et al., 2012). Dynamic light scattering experiments were carried out with a Malvern ZetaSizer, and dynamic mechanical analysis with a AR-2000 rheometer from TA-Instruments.

\section{RESULTS}

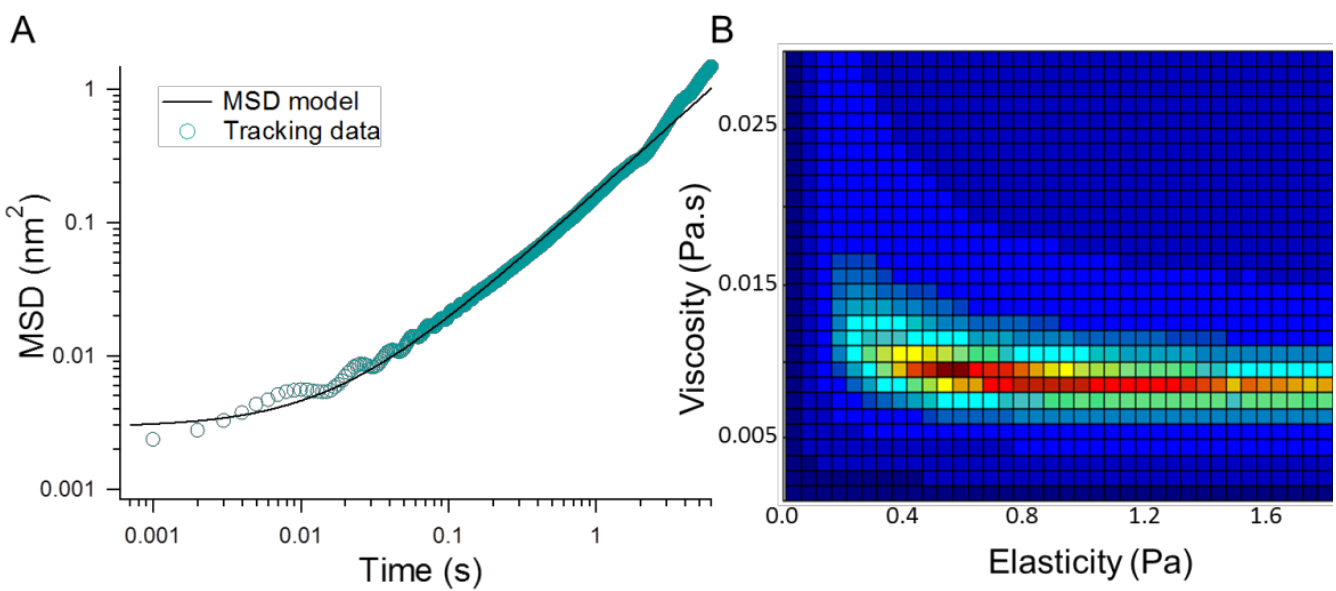

Figure 2: Microrheology for viscoelastic solution characterization. (A) Average MSD extracted from 5 particles diffusing in a solution containing (m:w) 15\% PVP $40 \mathrm{KDa}$ (blue dataset), and the fit with the single relaxation time Maxwell fluid model (in black). (B) The plot shows the inverse of the residue for the dataset shown in (A) as a function of the viscosity $\eta$ and the elastic modulus E.

\section{Viscosity, elasticity, and electrophoretic measurements}

The static viscosity $\eta$ and relaxation time $\tau$ of the polymer solutions were determined with particle-tracking microrheology (Mason et al., 1997) assuming the fluid behaved as a Maxwell fluid. We monitored the mean square displacement (MSD) of $1 \mu \mathrm{m}$ fluorescent particles, which was characterized by a linear slope in the longtime limit characteristic of Brownian diffusion (Fig. 2A). In the short-time limit, the MSD tended to reach a plateau that reflected the existence of the elastic properties of the liquid (van Zanten and Rufener, 2000). We minimized the residue between the experimental MSD and that predicted from the Maxwell model using the 
fluid viscosity $\eta$ and elastic modulus $E$ as adjustable parameters (Fig. 2B). Note that the fluid relaxation time $\tau$ is $\eta / E$. The viscosity was estimated with a better precision than the elasticity, as can be judged from the elongated region along the $x$-axis of the parameter space with minimal residual (Fig. 2B). We checked the consistency of our measurements by (i) assaying the viscosity of the same solution with dynamic light scattering, and (ii) performing dynamic mechanical analysis to estimate the elasticity. The value of the viscosity and elasticity were similar within an error of $\sim 20 \%$ (not shown).

Table 1 summarizes the fluid viscosity and the relaxation time scale. The viscosity expectedly increased with the concentration and molecular weight of PVP with a range of variation between 6 and $31 \mathrm{mPa}$.s. Most experiments have been carried out with a viscosity lower than $15 \mathrm{mPa} . \mathrm{s}$, because better separation performances were achieved with the pressure and voltage operating conditions of our set up. The electrophoretic mobility $\left(\mu_{e}\right)$ was determined using a 300 bp DNA strand, which was concentrated by electro-hydrodynamic actuation in order to obtain a thin and detectable band. This band was then conveyed by pure electrophoresis in the constriction for different viscoelastic solutions. The electrophoretic mobility was calculated using the velocity of the band at the apex of the system, measured form the obtained videos (Table 1).

Table 1: Viscosity, Elastic modulus, and relaxation time and electrophoretic mobility of different polymer solutions inferred from micro-rheology techniques. The stiffness corresponds to the parameter defined in Eq. (7), and it is estimated for the conditions of maximal resolution for the band of $600 \mathrm{bp}$. The constant $K$ ' is the adjustable parameter of our model, as defined in Eq. (7).

\begin{tabular}{ccccccc}
\hline (MW) & $($ wt \%) & $\begin{array}{c}\text { Viscosity } \eta \\
(\mathrm{mPa} . \mathrm{s})\end{array}$ & $\begin{array}{c}\text { Relaxation } \\
\text { time }(\mathrm{ms})\end{array}$ & $\begin{array}{c}\text { Electrophoretic } \\
\text { Mobility }\left(\mu_{e}\right) \\
10^{-5}\left(\mathrm{~cm}^{2} \cdot \mathrm{V}^{-1} \cdot \mathrm{s}^{-1}\right)\end{array}$ & $\begin{array}{c}k \\
\left(10^{-3} \mathrm{pN} . \mathrm{nm}^{-1}\right)\end{array}$ & $\begin{array}{c}K^{\prime} \\
\left(10^{-1}\right)\end{array}$ \\
\hline & 10 & $6.0 \pm 1.2$ & $15.0 \pm 1.5$ & $6.1 \pm 0.1$ & $4.8 \pm 0.1$ & $0.13 \pm 0.01$ \\
PVP 40 kDa & 13 & $8.0 \pm 1.3$ & $16.0 \pm 1.6$ & $4.5 \pm 0.1$ & $6.4 \pm 0.1$ & $0.75 \pm 0.01$ \\
& 15 & $9.5 \pm 1.3$ & $14.2 \pm 1.4$ & $3.3 \pm 0.1$ & $3.4 \pm 0.1$ & $1.02 \pm 0.01$ \\
& 20 & $12.0 \pm 1.2$ & $14.1 \pm 1.4$ & $3.0 \pm 0.1$ & $6.7 \pm 0.1$ & $1.89 \pm 0.01$ \\
& 2 & $10.0 \pm 1.2$ & $15.3 \pm 1.5$ & $2.4 \pm 0.1$ & $3.6 \pm 0.1$ & $2.56 \pm 0.01$ \\
PVP 1.3 MDa & 3 & $15 \pm 2$ & $17.5 \pm 1.8$ & $10.5 \pm 1.5$ & $8.3 \pm 0.1$ & $4.32 \pm 0.01$ \\
& 4 & $21 \pm 1$ & $30 \pm 3$ & $9.5 \pm 1.0$ & $53.0 \pm 0.1$ & $7.85 \pm 0.01$ \\
& 5 & $31 \pm 2$ & $43 \pm 3$ & $8.3 \pm 0.7$ & $43.0 \pm 0.1$ & $8.65 \pm 0.01$ \\
\hline
\end{tabular}

\section{Separation experiments with the 50 bp DNA ladder}

We carried out a set of separation experiments using a DNA ladder containing 11 bands in the range 50 to $1000 \mathrm{bp}$. We tested a chip with a power-law $v s$. a linear geometry of $m=2.5 \mathrm{vs} . m=1$, respectively, and used different formulations of PVP. As an example, we report fluorescence micrographs using a pressure of 7 bar and a tension of $300 \mathrm{~V}$ in both directions using the chip with a power-law vs. linear funnel (Fig. 1B). Note that the electrophoretic velocity always represented $2-5 \%$ of the maximum flow velocity. The plots of the intensity distribution along the symmetry axis of the channel indicate that the bands with the bands in the range 50 to 300 bp are only detectable in the power-law constriction (Fig. 1C). Whenever a band was clearly detected, we reported its position, width and resolution as well as chip geometry, fluid properties, and actuation parameters in a database for subsequent analysis.

\section{Validation of the model}

i. $\quad$ Position of the DNA bands (Equation 12)

We first focused on the position $x_{600}$ of the band of the $600 \mathrm{bp}$. Following the prediction of Eq. (12), $x_{600}$ was raised to the power exponent $2 m$ and plotted as a function of the ratio $V_{e_{\max }}{ }^{3} / V_{p_{\max }}$ (Fig. 3A\&B). In five different formulations of PVP $40 \mathrm{kDa}$ and for two funnel geometries, we obtained linear trends associated to an excellent Pearson correlation coefficient greater than 0.95 . Given the linear relationship between $V_{e_{\max }}$ or $V_{p_{\max }}$ and the voltage or pressure, respectively, this correlation was equally even with the nominal input parameters of the experiment. Consequently, the response of DNA as a function of the constriction geometry or the actuation parameters is assessed with our model. Furthermore, we noticed that the slopes of the different 
datasets steepened with the concentration of PVP in both funnel shapes (Fig. 3A\&B). Because the prefactor linking $x_{600}{ }^{2 m}$ to $V_{e_{\max }}{ }^{3} / V_{p_{\max }}$ in Eq. (12) is determined by the fluid properties through the product $\eta \tau$, and the viscosity increases with the concentration of $40 \mathrm{kDa}$ PVP while the relaxation time is roughly constant (Table 1), the result appears to be qualitatively coherent with our model.

More quantitatively, we rescaled our data using the product $\eta \tau$ and $\mathrm{K}$ ' which depends on PVP concentration $C_{P V P}$ as input parameters. The datasets collapsed on to one master response for both geometries with a prefactor of $C_{P V P}{ }^{2.5} \eta \tau$ (Fig. 3C\&D). We note that the same response was obtained for the set of formulations of PVP 1.3 $\mathrm{MDa}$ (Supplementary Fig. S2 and S3).
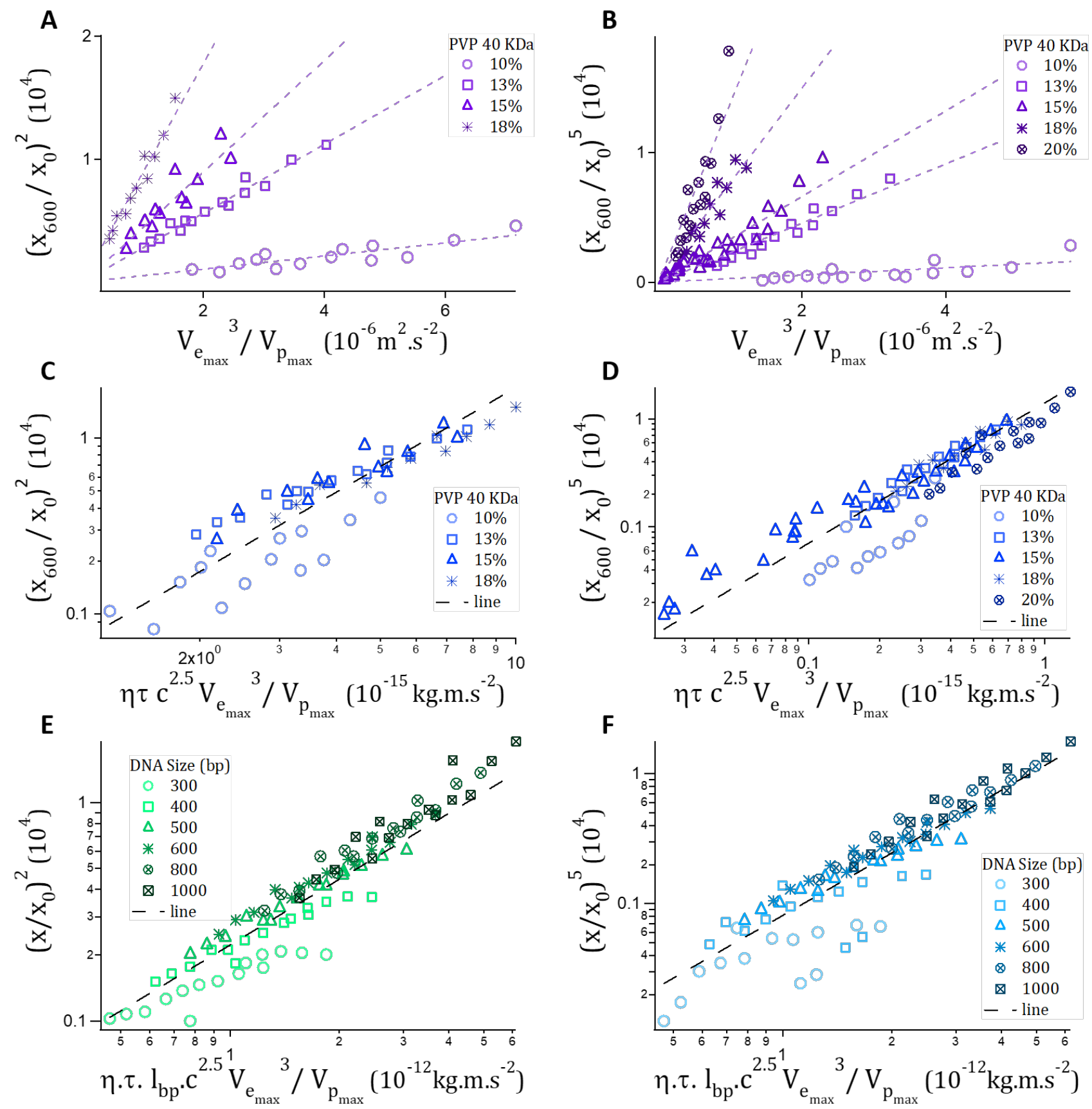

Figure 3: Analysis of the band position. (A) The position $x_{600}$ of the band of $600 \mathrm{bp}$ raised to the power $2 \mathrm{~m}$ with $\mathrm{m}=1$ is plotted as a function of $V_{e_{\max }}{ }^{3} / V_{p_{\max }}$. We use a set of $40 \mathrm{kDa} P V P$ concentrations, as indicated in the legends.(B) Same plot as in (A) with $m=2.5$. (C-D) Every dataset in $(A)$ and $(B)$ are normalized with the product $C_{P V P}{ }^{2.5} \eta \tau$ in order to fall on to a master response. $(\boldsymbol{E}-\boldsymbol{F})$ The position $x$ of the bands of 300 to $1000 \mathrm{bp}$ are plotted as a function of $V_{e_{\max }}{ }^{3} / V_{p_{\text {max }}}$ rescaled with $C_{P V P}{ }^{2.5} \eta \tau$ and the DNA contour length $l_{b p}$ for the linear and power law geometry, respectively. 
Finally, we studied the position of the DNA bands in the range 300 to $1000 \mathrm{bp}$, which were rescaled to the power $2 m$ and plotted as a function of $V_{e_{\max }}{ }^{3} / V_{p_{\max }}$ (Fig. 3E-F). For each DNA size, a linear trend was obtained, and the resulting slopes appeared to steepen with DNA MW (not shown). According to our model in Eq. (12), the slopes are dictated by the effective size $a$ of the molecules. The datasets collapsed on to a master curve for $a \sim l_{b p}$ (length in base pair) for both linear and power-law geometries (Fig. 3E-F). For small molecules, the size of which is comparable to the Kuhn length of $300 \mathrm{bp}$, this result is consistent with a rod-like behavior, for which the hydrodynamic radius is dictated by the contour length. For longer chains of $\sim 1000 \mathrm{bp}$, i.e. composed of $\sim 3$ Kuhn segments, we suggest that the Rouse model applies because hydrodynamic interactions are screened out due to the presence of high amounts of neutral polymers. The total transverse force acting on DNA can thus be decomposed into the sum of elementary forces undergone by each Kuhn segment, meaning that the term $a$ can be replaced by $N a$ in Eq. (12). Consequently the average position $\varepsilon$ is expected to be determined by the contour length of the molecule.

\section{ii. $\quad$ Breadth of the DNA bands (Equation 17)}

We analyzed the breadth of a $600 \mathrm{bp}$ band in different concentrations of PVP $40 \mathrm{kDa}$. Our model predicts the amplitude of the breadth to be proportional to $\left(V_{e_{\max }} / V_{p_{\max }}\right)^{3 / 2}$. This prediction is confirmed by our observations, as shown by the average Pearson correlation coefficient of 0.75 measured for 9 different PVP formulations (four of which are shown in Fig. 4A). Furthermore, we could collapse the breadth data on a master curve using the prefactor of $\sqrt{\eta / \tau}$ expected from Eq. (17) (Fig. 4A-B).

A

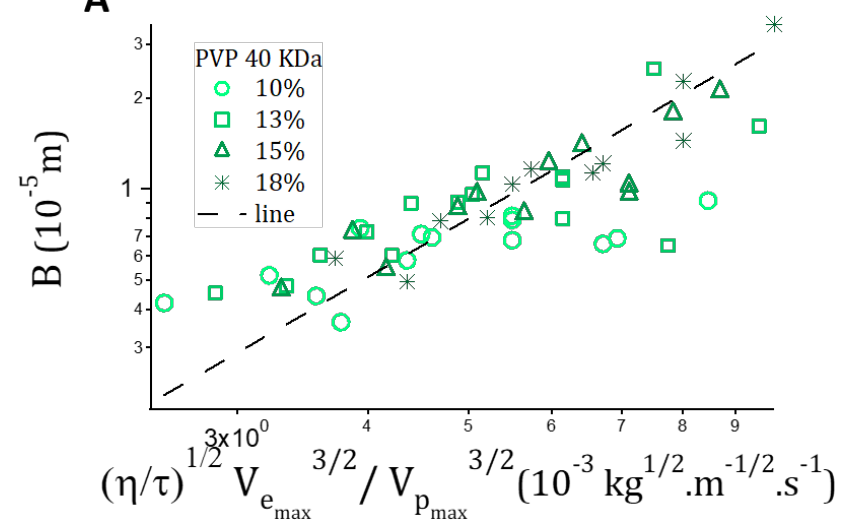

B

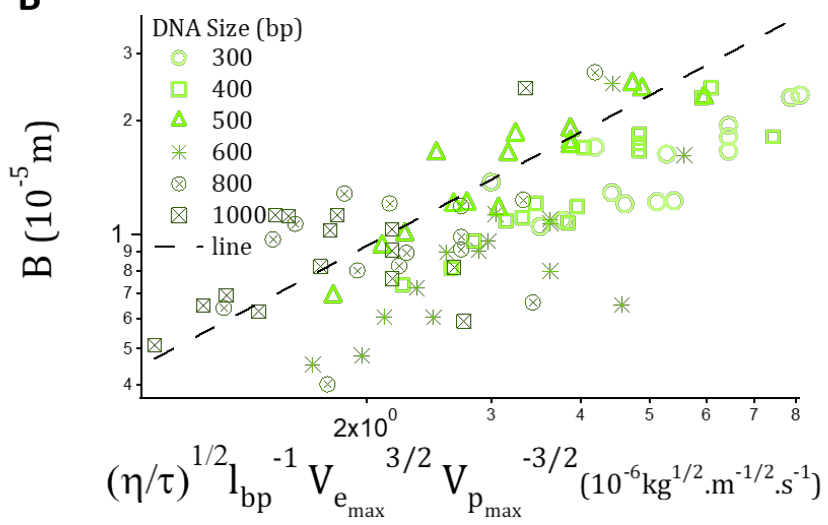

Figure 4: Analysis of the breadth. (A) The breadth $B$ of the $600 \mathrm{bp}$ band is reported for four different concentrations of PVP $40 \mathrm{kDa}$, as recapped by the ratio $\sqrt{\eta / \tau}\left(V_{e_{\max }} / V_{p_{\max }}\right)^{3 / 2}$.(B) The breadth of different DNA bands (300-1000 bp), in PVP 40 KDa $13 \%$, is plotted with the inverse of $l_{b p}$.

The model predicts a proportionality of the breadth with the square root of DNA size (see Eq. (17)). This fact was however incongruous with the rescaling of the data where the breadth decreased with the increase in the DNA MW. (Fig. 4B). Consequently, our model captures the physics of DNA band broadening in terms of electro-hydrodynamic actuation, but fails to describe the dependence of the breadth on the DNA MW. We suggest that this inconsistency stems from the deformability of DNA molecules near the wall. Transverse viscoelastic forces compress the molecules, which cannot be considered as a spherical tracers of dimension $\mathrm{a} \sim l_{b p}$. We believe that the physical size for vertical fluctuations is overestimated in our model, and propose to study this physics with Brownian dynamics simulations (manuscript in preparation).

\section{iii. $\quad$ Resolution per base pair (Equation 20)}

Using our measurements of the position and width of the different bands, we computed the resolution per base pair $\left(R_{b p}\right)$. Because our model described the position of the band with an excellent accuracy, but showed some inconsistencies for the breadth of the bands, we have either considered the detailed expression of $R_{b p}$ (Eq. (20)), or assumed for the sake of simplification that the breadth was constant in Eq. (19). It is noteworthy that $R_{b p}$ has a low range of variation of a factor of two (upper panel in Fig. 5A), so the discrimination between different models by scaling analysis is not very strong. 
We reported $R_{b p}$ for different PVP $40 \mathrm{kDa}$ concentrations and for DNA bands of 300 to $800 \mathrm{bp}$ in a powerlaw geometry of $m=2.5$ using the scaling prediction of Eq. (19) and obtained some degree of correlation associated to a Pearson coefficient of 0.65 (upper panel in Fig. 5A). Note that the same trend was obtained in PVP 1.3 MDa (supplementary Fig. S4). We tried to carry out the same analysis using Eq. (20), but the correlation decreased significantly below 0.2 (not shown). Consequently, the position of the bands, which constitutes the governing parameter of Eq. (19), appears to dictate the resolution of separation experiments carried out by $\mu$ LAS.

The relevance of our conclusion can be readily judged by performing separation experiments with two electric fields of 250 and $380 \mathrm{~V}$ and the same actuation pressure as well as $40 \mathrm{kDa}$ PVP solution at $18 \%$ (lower and upper panel of Fig. 6B-C, respectively). The DNA bands of 500 to $1000 \mathrm{bp}$ are not base-line resolved for the lower voltage, but they can be clearly identified at high voltage, demonstrating the increase in resolution with the electric field.

A
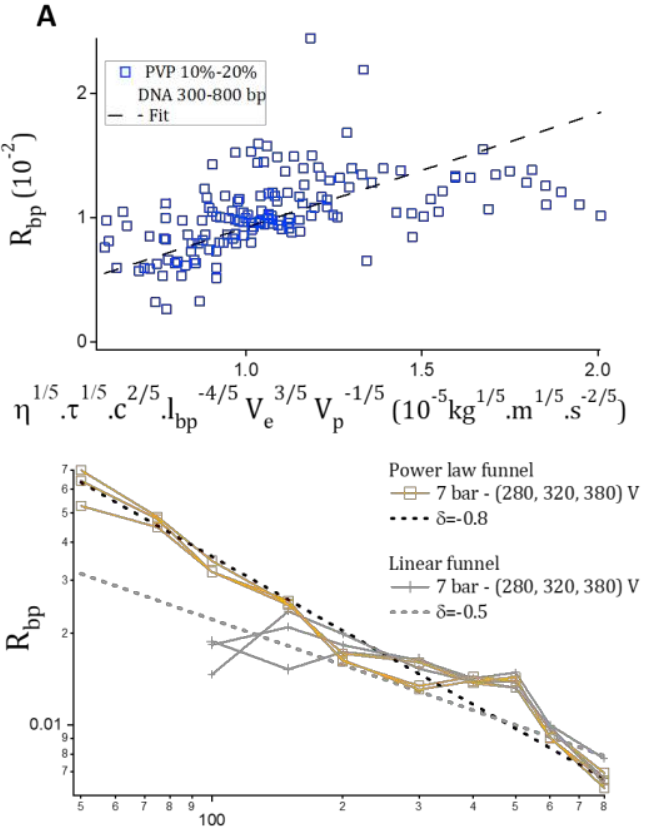

DNA Fragment Size (bp)
B
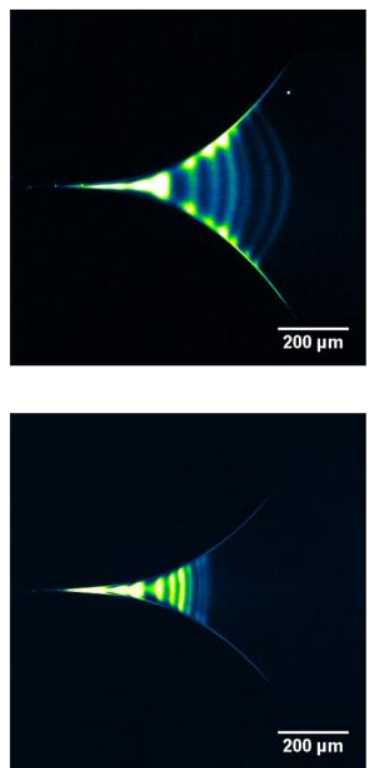

C
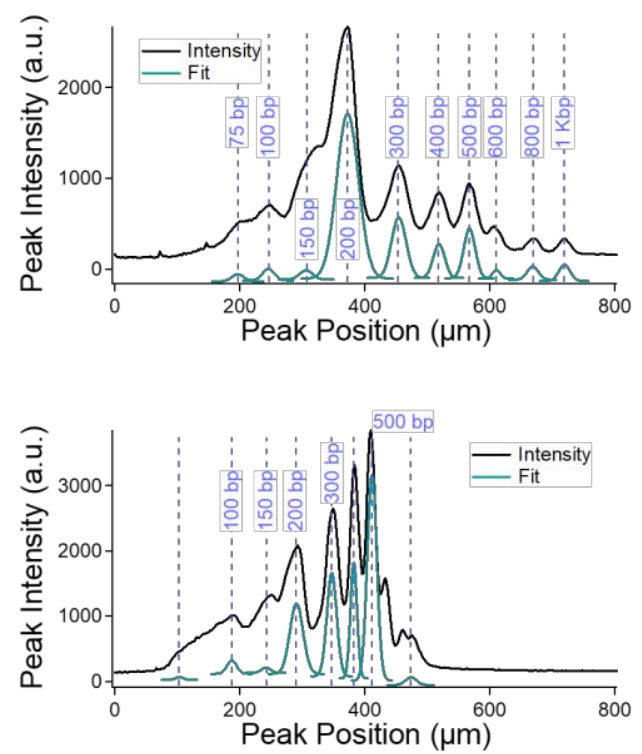

Figure 5: Model-data analysis of the $\boldsymbol{R}_{b p}$. (A) The upper panel shows $R_{b p}$ for DNA bands of 300 to 1000 bp in different concentrations of 10 to $20 \%$ (m:w) of PVP $40 \mathrm{kDa}$ as a function of the normalization factor derived in Eq. (19). The lower panel corresponds to $R_{b p}$ for a power-law and a linear geometry as a function of DNA MW (orange and gray datasets, respectively). We use three different settings for the voltage with the same pressure and PVP concentration (18\% PVP 40 $K D a$ ). The dashed lines correspond to the predictions of the model in Eq.(19). (B) The fluorescence micrographs of a 50 bp DNA ladder separated in 18\% (m:w) $40 \mathrm{kDa}$ PVP at a hydrodynamic speed of $146 \mathrm{~mm} / \mathrm{s}$ under electrophoretic speeds of

5.4 and $3.5 \mathrm{~mm} / \mathrm{s}$ (upper and lower panel, respectively).(C) The respective fluorescence intensity profiles of the micrographs in $(B)$, the profiles are fitted with a noise value of 7 and a smoothing of 3 .

\section{Definition of optimal separation settings}

As noted in Fig. 1, the detection of low MW fragments of 75 to $300 \mathrm{bp}$ is straightforward in power-law but impossible in linear funnels. In fact, we systematically observed that power-law geometries are superior to linear ones for low MW DNA separation whatever the viscoelastic fluid composition (not shown). This result is readily explained by the fact that the bands tend to mix and overlap near the funnel of the linear geometry due to the overly sharp variation of the electric and hydrodynamic velocities. Moreover, we computed $R_{b p}$ as a function of DNA size for different actuation conditions in the same PVP solution (40 kDa 18\%) and for two geometries (Fig. 7). Eq. (19) predicts that the $R_{b p}$ should vary with particle diameter $a$ following a power exponent $\delta=$ $1 / 2 m-1$ with $m$ the power law exponent of the funnel geometry. Because we showed that $a$ varies linearly with DNA MW, we expect $R_{b p}$ to vary with DNA MW with an exponent of -0.8 or -0.5 for $m=2.5$ or $m=1$, respectively. This trend is qualitatively confirmed by our measurements reported in the lower panel of Fig. 5A 
together with the two scaling responses represented by dashed lines.

Notably, the maximal $R_{b p}$ of 0.07 achieved for the DNA bands of 50 to $75 \mathrm{bp}$ in the power-law funnels corresponds to a Resolution length (RSL) of $14 \mathrm{bp}$. By scrambling our data, we obtain a maximal RSL of $\sim 7 \mathrm{bp}$ for a 50 bp DNA fragment with 13\% PVP $40 \mathrm{kDa}$ (Supplementary Fig. S5). From Fig. S5 and Table 1 we can hint that to get good separation under the actuation parameters of 0.1 to 10 bar and 5 to $400 \mathrm{~V}$, we should use solutions with viscosities spanning 8 to $15 \mathrm{mPa}$.s. Other formulations associated to high proportions of PVP will lead to slow hydrodynamic flows that are insufficient to trap $50 \mathrm{bp}$ fragments. Conversely, the use of low proportion of PVP, e.g. 10\% PVP $40 \mathrm{kDa}$, reduces the amplitude of viscoelastic forces (Table 1, see more below on the amplitude factor $K^{\prime}$ ) and forbids the trapping of low MW DNA molecules. This discussion thus shows that the viscoelastic properties of the polymer solution are important parameters in defining the separation performance. Since the RSL defines the minimum genomic distance that can be resolved, we conclude that the technology is relevant for conventional sizing with residual concentrations of DNA (Andriamanampisoa et al., 2018), but not for sequencing applications.

\section{CONCLUSION}

Altogether, we develop an analytical model of DNA separation by $\mu$ LAS technology and validate it with experiments. This first step of the model consists in calculating the expression of the transverse viscoelastic force exerted on spherical objects. We then use this force to predict the position, breadth and resolution of DNA bands in the range 50 to $1000 \mathrm{bp}$. Our model is in very good agreement with our data, allowing us to define optimal conditions for $\mu$ LAS operations. We establish that $\mu$ LAS achieves better performances in funnels with power-law geometries, and achieve optimal separation lengths of $7 \mathrm{bp}$ for a fragment of 50 to $100 \mathrm{bp}$. Our $\mathrm{Si} /$ glass technology is not yet capable of reaching single base pair resolution essentially due to limitations in the applied voltage and the associated silicon dioxide breakdown tension of $400 \mathrm{~V}$ given our oxide layer thickness of $\sim 250 \mathrm{~nm}$. Because the bonding of silicon chip is degraded with thicker oxide layers (not shown), we suggest to further enhance the separation performances by switching to a glass/glass technology. By increasing the electric field, we anticipate that the position of low MW DNA bands could be set further away from the constriction where their breadth would be narrower. By fine tuning the amplitude of the pressure and tension, we may then be able to target single base pair separations.

In contrast to conventional DNA separation depending on electrophoresis, which is based on DNA migration properties through a porous material, $\mu$ LAS involves transverse forces that convey the molecules in an elastic trap, the stiffness of which is defined by the product of the local maximum hydrodynamic velocity, electrophoretic velocity, and particle diameter (Eq. (7)). Because conservation laws apply for the electric and hydrodynamic fields, the stiffness is modulated spatially in the funnel over a broad range of $(800 / 5)^{2} \sim 25,000$, allowing us to easily identify actuation conditions for DNA trapping. Focusing on the fragment of $600 \mathrm{bp}$, we could evaluate the stiffness of the trap associated to conditions of highest separation resolution (last column of Table 1). The stiffness in the range 1 to $1010^{-3} \mathrm{pN} / \mathrm{nm}$ is therefore qualitatively comparable to that exerted by magnetic tweezers for single molecule manipulation (Neuman and Nagy, 2008).

Moreover our model and the associated rescaling of the data provide a unique solution to measure the amplitude of viscoelastic forces. Indeed, the slope of the data plotted in Fig. 3 is proportional to $K^{\prime}$, the constant that characterizes the amplitude of transverse viscoelastic forces (Ho and Leal, 1976). $K^{\prime}$ appeared to be on the order of $10^{-1}$ (Table 1). Given that its value is typically an order of magnitude greater than $K$ as defined in Eq. (1) (see Eq. 7), we deduce that $K$ is $\sim 10^{-2}$. The prediction of Ho and Leal (Ho and Leal, 1976) that $K$ is comprised between 1 and the Reynolds number of $\sim 10^{-4}$ in our experimental settings is therefore confirmed. This estimate in turn strengthens the consistency of our model, and even suggests that our approach to characterize viscoelastic liquids may prove useful for other microfluidic technologies (Yuan et al., 2018).

\section{ACKNOWLEDGEMENTS}

The authors are grateful to Rémi Malbec for his support to set up the technology. This work was partly supported by the LAAS-CNRS micro and nano-technologies platform, member of the French RENATECH network. B. C. PhD fellowship was funded by the "Ligue contre le Cancer". This work was partly supported by the project "Gene Extractor" from Région Midi-Pyrénées, and the ANR grant $\mu$ LAS (ANR-16-CE18-0028-01). 
DECLARATION OF COMPETING INTEREST

The authors have no competing interests to declare. 


\section{REFERENCES}

Albarghouthi, M.N., Barron, A.E., 2000. Polymeric matrices for DNA sequencing by capillary electrophoresis. Electrophoresis 21, 4096-4111.

Andriamanampisoa, C.-L., Bancaud, A., Boutonnet-Rodat, A., Didelot, A., Fabre, J., Fina, F., Garlan, F., Garrigou, S., Gaudy, C., Ginot, F., Henaff, D., Laurent-Puig, P., Morin, A., Picot, V., Saias, L., Taly, V., Tomasini, P., Zaanan, A., 2018. BIABooster: On-line DNA concentration and size profiling with a limit of detection of $10 \mathrm{fg} / \mu \mathrm{L}$. Application to high-sensitivity characterization of circulating cell-free DNA. Anal. Chem. https://doi.org/10.1021/acs.analchem.7b04034

Astorga-Wells, J., Vollmer, S., Bergman, T., Jörnvall, H., 2007. Formation of stable stacking zones in a flow stream for sample immobilization in microfluidic systems. Anal. Chem. 79, 1057-1063. https://doi.org/10.1021/ac061699f

Astorga-Wells, J., Vollmer, S., Tryggvason, S., Bergman, T., Jörnvall, H., 2005. Microfluidic Electrocapture for Separation of Peptides. Anal. Chem. 77, 7131-7136. https://doi.org/10.1021/ac050931z

D’Avino, G., Greco, F., Maffettone, P.L., 2017. Particle Migration due to Viscoelasticity of the Suspending Liquid and Its Relevance in Microfluidic Devices. Annu. Rev. Fluid Mech. 49, 341-360. https://doi.org/10.1146/annurev-fluid-010816-060150

D’Avino, G., Romeo, G., Villone, M.M., Greco, F., Netti, P.A., Maffettone, P.L., 2012. Single line particle focusing induced by viscoelasticity of the suspending liquid: theory, experiments and simulations to design a micropipe flow-focuser. Lab. Chip 12, 1638. https://doi.org/10.1039/c2lc21154h

Ge, Z., Wang, W., Yang, C., 2015. Rapid concentration of deoxyribonucleic acid via Joule heating induced temperature gradient focusing in poly-dimethylsiloxane microfluidic channel. Anal. Chim. Acta 858, 91-97.

Heller, C., 2001. Principles of DNA separation with capillary electrophoresis. ELECTROPHORESIS 22, $629-$ 643. https://doi.org/10.1002/1522-2683(200102)22:4<629::AID-ELPS629>3.0.CO;2-S

Ho, B.P., Leal, L.G., 1976. Migration of rigid spheres in a two-dimensional unidirectional shear flow of a second-ordre fluid. J Fluid Mech 76, 783-799.

Kaneta, T., Ueda, T., Hata, K., Imasaka, T., 2006. Suppression of electroosmotic flow and its application to determination of electrophoretic mobilities in a poly(vinylpyrrolidone)-coated capillary. J. Chromatogr. A 1106, 52-55. https://doi.org/10.1016/j.chroma.2005.08.062

Kuo, I.-T., Chiu, T.-C., Chang, H.-T., 2003. On-column concentration and separation of double-stranded DNA by gradient capillary electrophoresis. Electrophoresis 24, 3339-3347. https://doi.org/10.1002/elps.200305603

Leshansky, A.M., Bransky, A., Korin, N., Dinnar, U., 2007. Tunable Nonlinear Viscoelastic "Focusing" in a Microfluidic Device. Phys Rev Lett 98, 234501.

Lettieri, G.-L., Dodge, A., Boer, G., Rooij, N.F. de, Verpoorte, E., 2003. A novel microfluidic concept for bioanalysis using freely moving beads trapped in recirculating flows. Lab. Chip 3, 34-39. https://doi.org/10.1039/B211869F

Liu, P., Mathies, R.A., 2009. Integrated microfluidic systems for high-performance genetic analysis. Trends Biotechnol. 27, 572-581. https://doi.org/10.1016/j.tibtech.2009.07.002

Mason, T.G., Ganesan, K., Van Zanten, J.H., Wirtz, D., Kuo, S.C., 1997. Particle tracking microrheology of complex fluids. Phys. Rev. Lett. 79, 3282.

Neuman, K.C., Nagy, A., 2008. Single-molecule force spectroscopy: optical tweezers, magnetic tweezers and atomic force microscopy. Nat. Methods 5, 491-505. https://doi.org/10.1038/nmeth.1218

Paegel, B.M., Blazej, R.G., Mathies, R.A., 2003. Microfluidic devices for DNA sequencing: sample preparation and electrophoretic analysis. Curr. Opin. Biotechnol. 14, 42-50.

Park, S.-R., Swerdlow, H., 2003. Concentration of DNA in a Flowing Stream for High-Sensitivity Capillary Electrophoresis. Anal. Chem. 75, 4467-4474. https://doi.org/10.1021/ac034209h

Ranchon, H., Malbec, R., Picot, V., Boutonnet, A., Terrapanich, P., Joseph, P., Leïchlé, T., Bancaud, A., 2016. DNA separation and enrichment using electro-hydrodynamic bidirectional flows in viscoelastic liquids. Lab Chip 16, 1243-1253. https://doi.org/10.1039/C5LC01465D

Ranchon, H., Picot, V., Bancaud, A., 2015. Metrology of confined flows using wide field nanoparticle velocimetry. Sci. Rep. 5. https://doi.org/10.1038/srep10128

Ross, D., Locascio, L.E., 2002. Microfluidic temperature gradient focusing. Anal. Chem. 74, 2556-2564.

Sambrook, J., Fritsch, E.F., Maniatis, T., 1989. Molecular cloning: a laboratory manual. Mol. Cloning Lab. 
Man.

Simpson, S.L., Quirino, J.P., Terabe, S., 2008. On-line sample preconcentration in capillary electrophoresis: Fundamentals and applications. J. Chromatogr. A 1184, 504-541.

Tinevez, J.-Y., Perry, N., Schindelin, J., Hoopes, G.M., Reynolds, G.D., Laplantine, E., Bednarek, S.Y., Shorte, S.L., Eliceiri, K.W., 2017. TrackMate: An open and extensible platform for single-particle tracking. Methods 115, 80-90. https://doi.org/10.1016/j.ymeth.2016.09.016

van Zanten, J.H., Rufener, K.P., 2000. Brownian motion in a single relaxation time Maxwell fluid. Phys. Rev. E 62, 5389-5396. https://doi.org/10.1103/PhysRevE.62.5389

Yamaguchi, Y., Li, Z., Zhu, X., Liu, C., Zhang, D., Dou, X., 2015. Polyethylene Oxide (PEO) and Polyethylene Glycol (PEG) Polymer Sieving Matrix for RNA Capillary Electrophoresis. PloS One 10, e0123406.

Yuan, D., Zhao, Q., Yan, S., Tang, S.-Y., Alici, G., Zhang, J., Li, W., 2018. Recent progress of particle migration in viscoelastic fluids. Lab. Chip. https://doi.org/10.1039/C7LC01076A 


\section{Supplementary Material:}

\section{Supplementary Material 1:}

The hydraulic resistance of the chip:

The microchannel geometry, of rectangular cross section, with two different depths, $\mathrm{h}_{1}=16 \mu \mathrm{m}$ and $\mathrm{h}_{2}=2 \mu \mathrm{m}$ is represented in Fig. S6. Two different funnel geometries are present in the same chip, a linear shape of $m=1$ and a power-law shape of $m=2.5$ or 3 (Fig. S6 left and right), respectively. The width of the funnel at a position $w(x)$ is described by $w(x) / w_{0}=\left(x / x_{0}\right)^{m}+1$, where $w_{0}$ is the width of the constriction equal to $5 \mu \mathrm{m}$.

The hydraulic resistance $R_{h}$ of a rectangular cross-section is given by Eq. (21), where $L$ is the length of the channel.

$$
R_{h} \approx \frac{12 \eta L}{w h^{3}\left(1-\frac{0.63 h}{w}\right)}
$$

In the funnel region, the hydraulic resistance is given by:

$$
R_{h_{-} \text {funnel }} \approx \int \frac{12 \eta d x}{w_{0}\left[\left(x / x_{0}\right)^{m}+1\right] h_{2}^{3}-0.63 h_{2}^{4}}
$$

The hydrodynamic and electrophoretic velocities are derived using the nominal inputs of the experiments, namely the pressure and voltage, respectively.

Hydrodynamic speed:

The hydrodynamic velocity can be obtained by evaluating the flow rate $Q$ given by Eq. (23) where $\Delta P$ is the applied pressure difference and $R_{h_{-} \text {total }}$, the total hydraulic resistance of the channel:

$$
Q=\frac{\Delta \mathrm{P}}{R_{h_{-} \text {total }}}
$$

The maximum hydrodynamic velocity of the Poiseuille profile $V_{p_{0}}(x)$ in the region of interest, the funnel, can be derived from the flow rate using the following relation:

$$
Q=\frac{\Delta \mathrm{P}}{R_{h_{-} \text {total }}}=\frac{2}{3} h_{2} w_{0}\left[\left(x / x_{0}\right)^{m}+1\right] V_{p_{0}}(x)
$$

Therefore, the hydrodynamic velocity is proportional to the applied pressure difference as shown by Eq. (25):

$$
V_{p_{0}}(x)=\frac{3 \Delta P}{2 h_{2} w_{0}\left[\left(x / x_{0}\right)^{m}+1\right] R_{h_{-} \text {total }}}
$$

Electrophoretic speed:

The electrophoretic velocity $V_{e}$ can be derived from the electric field $E$ using Gauss' law of 
conservation of electric flux $\Phi$ along a cross-section $S$ (Eq.(26)) and the Gradient theorem (Eq. (27)):

$$
\begin{gathered}
\Phi=\oiint \vec{E} \cdot \overrightarrow{d S} \\
V(\vec{a})-V(\vec{b})=-\int_{a}^{b} \vec{E} \cdot \overrightarrow{d l}
\end{gathered}
$$

where $V(\vec{a})$ and $V(\vec{b})$ is the electric potential at points $a$ and $b$, respectively and $l$ the distance between the two points.

In the funnel region, the electric field at a given position in $\mathrm{KV} / \mathrm{m}$ is given by:

$$
E(x) \approx \frac{\alpha \Delta V}{w_{0}\left[\left(x / x_{0}\right)^{m}+1\right]}
$$

where $\alpha$ is a dimensionless constant that depends on the funnel geometry.

Finally, the electrophoretic speed, at a given position in the funnel, can be obtained by multiplying the electric field with the electrophoretic mobility of DNA in a given polymer solution (Table1).

$$
V_{e}(x)=\mu_{e} E(x)
$$




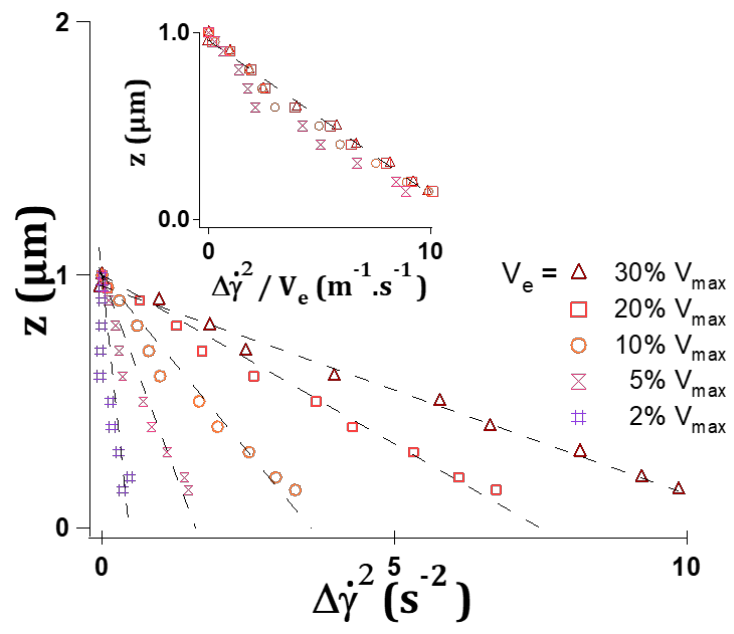

Figure S1: Transverse viscoelastic force finite element modeling. (B) Using 3D finite element modeling, we determine the difference in square of the shear rate on the upper and lower apexes of the particle, as reported in the $x$ axis of the graph. The channel height $h$, particle radius $a$, and hydrodynamic maximum speed $V_{p_{0}}$ are set to $2 \mu \mathrm{m}$, $50 \mathrm{~nm}$, and $75 \mu \mathrm{m} / \mathrm{s}$, respectively with different electrophoretic velocities, as indicated in the legend. The set of data can be rescaled with the electrophoretic velocity (inset). Dashed lines correspond to guides to the eye.

A

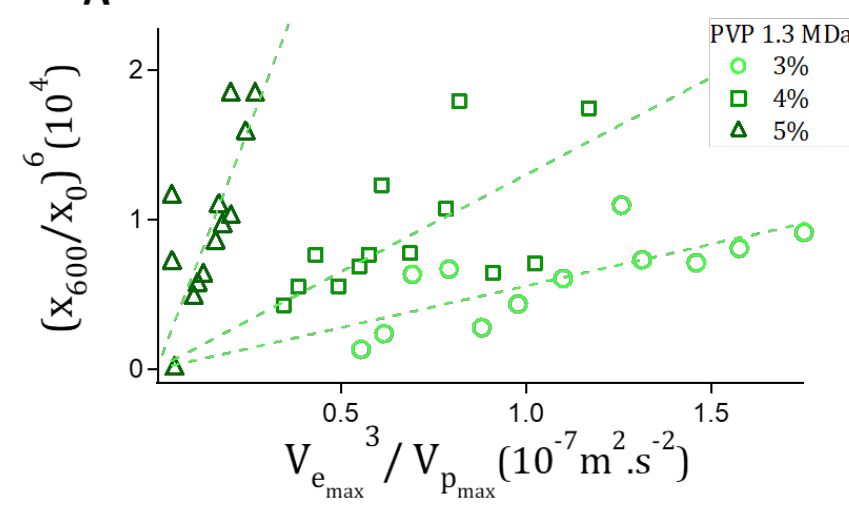

B

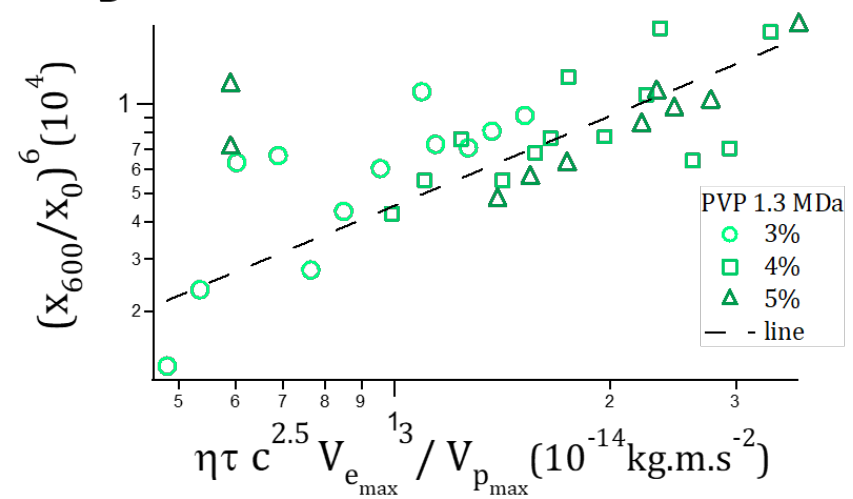

Figure S2: Analysis of the band position. (A) The position $x_{600}$ of the band of 600 bp raised to the power $2 \mathrm{~m}$ with $m=3$ is plotted as a function of $V_{e_{\max }}{ }^{3} / V_{p_{\max }}$. We use a set of $1.3 \mathrm{MDa}$ PVP concentrations, as indicated in the legends. (B) The datasets in (A) are normalized with the product $C_{P V P}{ }^{2.5} \eta \tau$ in order to fall on to a master response.

A

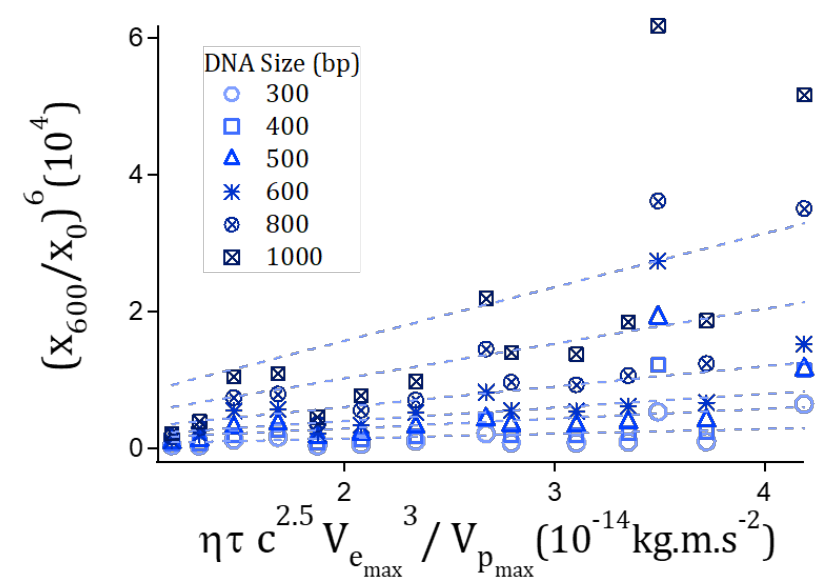

B

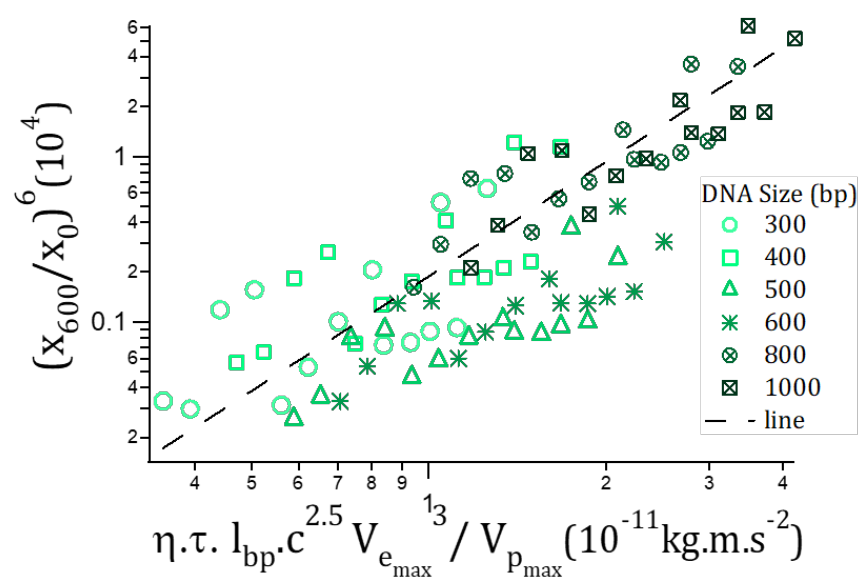

Figure S3: Size dependence of DNA band positions. (A) The plot represents the position of the bands of 300 to 1000 bp raised to the power $2 m$ with $m=3$ as a function of $V_{e_{\max }}{ }^{3} / V_{p_{\max }}$. (B) The same data is rescaled with 
the normalization factor $C_{P V P}{ }^{2.5} \eta \tau$ and DNA contour length $l_{b p}$ for the power law geometry.

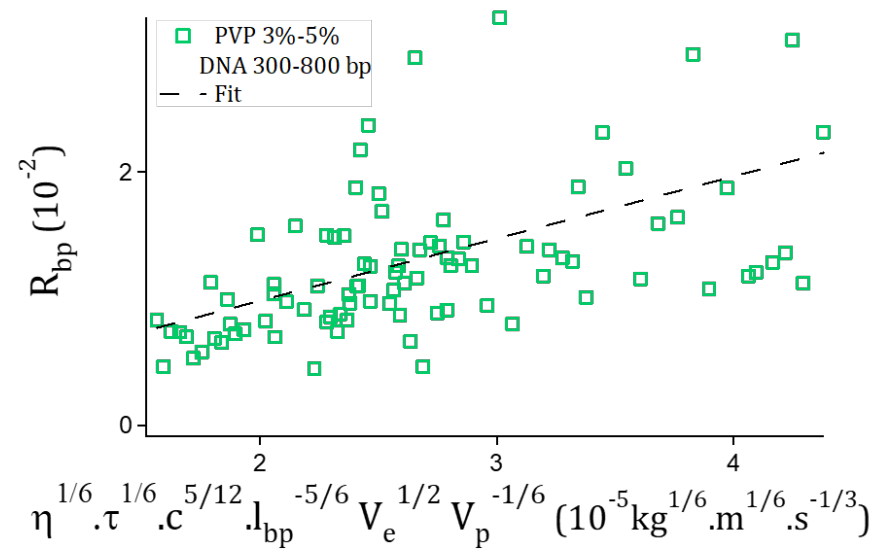

Figure S4: $\boldsymbol{R}_{b p}$ variation with DNA MW. The plot shows $R_{b p}$ for different DNA stands of 300 to $1000 \mathrm{bp}$, for a power-law geometry $(m=3)$. We use three different polymer concentrations (3\%, 4\% and 5\% PVP $1.3 \mathrm{MDa}$ ). 


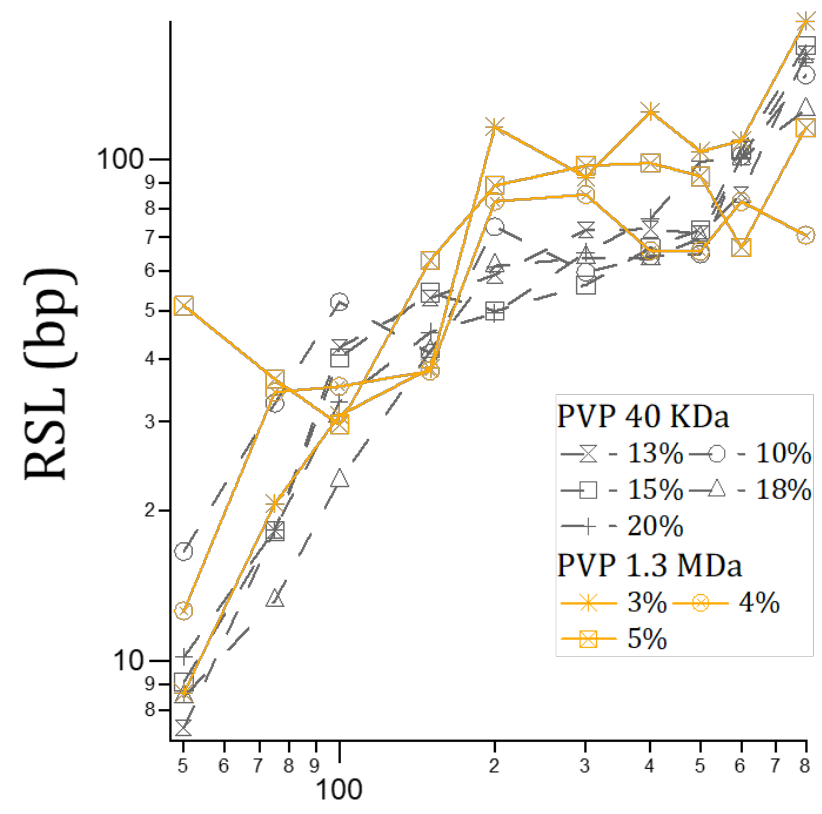

DNA Fragment Size (bp)

Figure S5: The RSL as a function of DNA size in different PVP solutions and concentrations. The graph shows the ability to reach 7 bp RSL for a 50 bp DNA band with PVP 40 KDa 13\%.

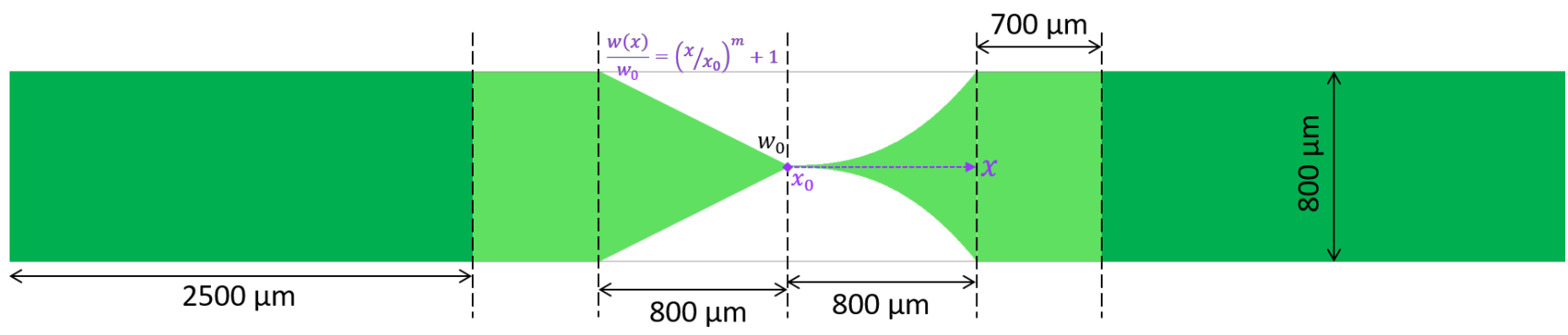

$\mathrm{h}_{1}=16 \mu \mathrm{m}$ $\mathrm{h}_{2}=2 \mu \mathrm{m}$

Figure S6: The microchannel geometry, of rectangular cross-section, containing two funnel shapes linear of $m=1$ and power-law of $m=2.5$ or 3, to the left and right, respectively. The channel has two depths $h_{1}=16 \mu \mathrm{m}$ and $h_{2}=2 \mu \mathrm{m}$ represented in dark and light green, respectively. 
Dear Editor,

Please find enclosed our manuscript entitled "Modeling of DNA transport in viscoelastic electro-hydrodynamic flows for enhanced size separation" by Chami et al., which we propose for publication in Biosensors and Bioelectronics as an article.

Recently, research on particle migration in non-Newtonian viscoelastic fluids has gained considerable attention. Three dimensional particle manipulation can be easily realized in simple channels without the need for any external force fields or complex microchannel structures. Hence, viscoelastic fluids offer a remarkable tool for molecular or cellular analysis. Yet, to the best of our knwoledge, analytical operations based on viscoelastic fluids have not been rigorously optimized due to the lack of models as well as the insufficient parametrization of fluid properties.

In this report, we wished to fill this gap by proposing a model of viscoelastic transport and by validating with experiments. Our research has been performed in the context of the $\mu \mathrm{LAS}$ technology for DNA separation, concentration, and analysis with high sensitivity (Ranchon, Lab Chip, 2016 \& Comtet, Anal Chem, 2018). The $\mu$ LAS technology is based on viscoelastic transverse migration using an hydrodynamic flow as well as an opposite electrophoretic force. Combining hydrodynamic first principles and statistical mechanics, we provide a quantitative model of DNA transport capable of predicting the device performance with the exclusive use of one adjustable parameter associated to the amplitude of transverse viscoelastic forces. Notably, this parameter of ubiquitous relevance for microfluidics with viscoelastic fluids is measured for the first time in microchannels and for biomolecules. The model proves to be in remarkable agreement with DNA separation experiments, and allows us to define optimal conditions that result in a maximal resolution length of $7 \mathrm{bp}$. We finally discuss the usefulness of our model for separation technologies involving viscoelastic liquids.

This paper therefore describes a model of viscoelastic transport and thoroughly compares its prediction to experiments that rely on state-of-the-art microfabrication and image processing. Hence, it fulfills the criteria of novelty and interdisciplinarity that are relevant for Biosensors and Bioelectronics.

With our best regards,

The authors 
PS: Word-count for the body of text $\sim 5400$ words and 6 displayed items List of suggested reviewers:

- Todd Squires, squires@engineering.ucsb.edu, University of California, Santa Barbara, USA

- Yoshinobu Baba, ca.u-ayogan.mehcpa@ttmyabab, Nagoya University, Japan

- Jason E. Butler, butler@che.ufl.edu, University of Florida, USA

- Weihua Li, weihuali@uow.edu.au, University of Wollongong, Australia

- Pier Luca Maffettone, pierluca.maffettone@unina.it, University of Naples Federico II, Italy 
Quantitative model of DNA transport in viscoelastic liquids

Transverse viscoelastic forces exerted on DNA are measured in situ

The model is in remarkable agreement with DNA separation experiments

Optimal settings for DNA separation are retrieved from the model 